\title{
Lensless metrology for semiconductor lithography at EUV
}

\author{
Iacopo Mochi, Dimitrios Kazazis, Li-Ting Tseng, Sara Fernandez, Rajendran Rajeev, Uldis \\ Locans, Atoosa Dejkameh, Ricarda Nebling, and Yasin Ekinci \\ Laboratory for Micro- and Nanotechnology, Paul Scherrer Institute, CH-5232 Villigen PSI, \\ Switzerland
}

\begin{abstract}
The production of modern semiconductor devices is based on photolithography, a process through which a pattern engraved on a mask is projected on a silicon wafer coated with a photosensitive material. In the past few decades, continuous technological progress in this field allowed the industry to follow Moore's law by reducing the size of the printed features. This was achieved by progressively increasing the numerical aperture of the projection system and reducing the wavelength. The latest lithography platforms for semiconductor manufacturing employ Extreme Ultra Violet (EUV) light at a wavelength of $13.5 \mathrm{~nm}$. The metrology for the optics and the components of such platforms is not fully mature yet. Specifically, the inspection of the EUV photomask is still an open issue as no commercial solutions are currently available. Here we describe a lensless approach to this problem, based on coherent diffraction imaging at EUV that overcomes the main technological issues linked to the conventional mask inspection approach.
\end{abstract}

Keywords: Extreme ultra violet, lensless imaging, defect inspection, semiconductor lithography.

\section{INTRODUCTION}

Semiconductor devices manufacturing is a growing industry with a massive impact on our society. In the last few decades the technological progress in this field has followed Moores law, almost doubling the density of transistors in integrated circuits every two years. The key technology behind this progress is photolithography, the process through which a semiconductor device layout is transferred from a master (mask) to a silicon wafer. As any light projection process, the resolution of photolithography is linked to the wavelength. Until recently, the shortest wavelength available for the production of semiconductor devices was Deep Ultra Violet (DUV, $\lambda=193 \mathrm{~nm}$ ), but in 2018, after many years of research and development, Extreme Ultra Violet (EUV, $\lambda=13.5 \mathrm{~nm}$ ) lithography made its appearance in semiconductor fabs.

EUV does not propagate in air and it is strongly absorbed by most materials. For this reason, EUV optics are made of multilayer-coated mirrors. This includes the mask carrying the pattern to be projected on the wafer. The mask consists of a multilayer-coated $15 \times 15 \times 6 \mathrm{~mm}$ quartz substrate with a patterned $70 \mathrm{~nm}$-thick absorber layer on top. A defect on the pattern or in the reflective substrate could be replicated on thousands of wafers, hence the necessity of accurate and reliable mask metrology. The relevant size of the defects scales down with the critical dimension of the pattern on the mask. Currently, absorber defects as small as $10 \mathrm{~nm}$ and substrate defects with a FWHM of $50 \mathrm{~nm}$ and a thickness of $0.6 \mathrm{~nm}$ can be critical for the manufacturing process. Mask inspection and mask review are two metrology processes required to mitigate the impact of defects on the production of semiconductor devices. The goal of mask inspection is to identify the location of potential defects on the mask and provide an initial classification of their size. The main challenge is to map the whole mask with a sufficient sensitivity in the shortest time possible. Note that detecting a $10 \mathrm{~nm}$ defect on a standard EUV mask is roughly equivalent to finding a missing brick in the whole state of New York. Once the defects have been identified, the review process allows to estimate their impact on the printed image. This step consists in reviewing the defects with the same illumination and imaging settings that will be used to project the mask onto the silicon wafer. It is worth noting that, unlike the verification of the features printed on the wafer or

Further author information: (Send correspondence to Iacopo Mochi.)

E-mail: iacopo.mochi@psi.ch

Modeling Aspects in Optical Metrology VII, edited by Bernd Bodermann, Karsten Frenner, Proc. of SPIE Vol. 11057, $1105703 \cdot$ @ 2019 SPIE · CCC code: 0277-786X/19/\$21 · doi: 10.1117/12.2534350 
the inspection of the manufactured semiconductor device, it is important to perform mask metrology with EUV light since defects may have a strongly wavelength-dependent behavior. ${ }^{1,2}$

Since the beginning of the interest of the semiconductor interest in EUV, the search for reliable and affordable metrology platforms has been frustrated by the high cost and complexity of the optics and by the high resolution and throughput requirements. As of today, there is only one commercial metrology platform for mask review, ${ }^{3}$ but no affordable solutions are yet available for EUV mask inspection. An alternative way to address this problem is to use a lensless approach based on coherent diffraction imaging (CDI) and remove the projection optics from the equation. The reflective-mode EUV mask scanning lensless imaging microscope (RESCAN) developed at the Paul Scherrer Institut, is one of the first attempts ${ }^{4}$ to use soft X-Ray ptychography for EUV mask inspection. In this paper, we will describe its working principle, its potential for metrology and the results of the most recent experiments performed to assess its sensitivity to EUV mask defects.

\section{COHERENT DIFFRACTION IMAGING}

Coherent diffraction imaging (CDI) is a method to reproduce an image of a sample from its diffraction. The general idea behind it is to reproduce computationally the effect of an optical imaging system. Most reconstruction techniques are based on an iterative procedure that starts from an initial guess of the electromagnetic field in the object plane. The light is propagated to the detector plane where the phase of the field is retained while the magnitude is substituted by the measured diffraction pattern. Finally the field is propagated back to the object plane where the current guess is updated. This procedure is iterated until convergence is reached. ${ }^{5}$ To avoid local minima or artifacts in the reconstruction procedure, it is generally necessary to enforce some constraint in the object plane, like the sample support shape.

Thanks to the increasing availability of computational power and the rapid development of coherent X-ray sources, CDI methods gained popularity in the recent years. In particular, CDI is often used at wavelengths for which optical components are complex or expensive, like the case of X-rays and EUV.

\subsection{Ptychography}

Among the various image reconstruction methods used in CDI, ptychography is one of the most interesting for its capability of reconstructing both the complex image of the sample and the illumination function. ${ }^{6}$ In a nutshell, ptychography consists of scanning a sample with a coherent light probe allowing some degree of overlap between the illuminated regions, recording the diffraction patterns in the far field region and using this information to retrieve a consistent estimate of the complex amplitude of the illumination probe and of the sample under investigation. As in other coherent diffraction imaging approaches, when the diffraction pattern is recorded, the phase information is lost, and it must be retrieved to reconstruct the sample image. In ptychography, the phase is obtained from the information redundancy generated by the overlap of the illuminated regions. The image reconstruction process can be regarded as a minimization problem subject to two constraints: the intensity distribution of the recorded diffraction patterns and the necessity of a unique object in the overlapping regions of the scan. Let $O(\vec{r})$ be the complex amplitude of the object and $P(\vec{r})$ the complex amplitude of the illumination probe expressed as a function of the position vector $\vec{r}$. The object is scanned in discrete steps under the probe and for each position a new object exit wave $\phi_{j}=O\left(\vec{r}+\overrightarrow{r_{j}}\right) P(\vec{r})$ is defined by the product of the object and the illumination probe. Each exit wave propagates to the detector plane where it takes the form: $\Phi_{j}\left(\overrightarrow{r^{\prime}}\right)$ :

$$
\phi_{j} \rightarrow F\left(\phi_{j}\right)=\Phi_{j}\left(\overrightarrow{r^{\prime}}\right)
$$

where $F$ is the appropriate propagation operator that depends on the optical configuration of the system. ${ }^{7}$ The complex intensity distribution on the detector plane can be expressed as:

$$
\Phi_{j}\left(\overrightarrow{r^{\prime}}\right)=A_{j}\left(\overrightarrow{r^{\prime}}\right), \mathrm{e}^{i \alpha_{j}\left(\vec{r}^{\prime}\right)}
$$

where $A_{j}$ is the field amplitude and $\alpha_{j}$ is the phase. Let $\tilde{A}_{j}$ be the measured diffraction amplitude distribution, the goal is to find the best combination of the functions $\tilde{O}(\vec{r})$ and $\tilde{P}(\vec{r})$ that satisfy the equation:

$$
F\left(\phi_{j}\right)=\tilde{A}_{j} \mathrm{e}^{i \alpha_{j}}
$$


for every scan position. This is equivalent to minimizing the functional:

$$
G(P, O)=\sum_{j}\left|F\left(O\left(\vec{r}+\overrightarrow{r_{j}}\right) P(\vec{r})\right)-\tilde{A}_{j} \mathrm{e}^{i \alpha_{j}}\right|
$$

This task is usually undertaken with iterative procedures..$^{7-10}$ For the current application, we implemented a method based on the difference map algorithm. ${ }^{6}$

\section{RESCAN}

The Reflective-mode EUV mask SCANning lensless microscope (RESCAN) is a synchrotron-based EUV imaging system dedicated to EUV mask inspection and review. RESCAN uses ptychography to reconstruct the complex amplitude of the EUV mask surface and detect deviations from the ideal reticle image. The optical layout of the system is shown in figure 1. The EUV beam from the synchrotron is focused by a toroidal mirror $\left(\mathrm{M}_{1}\right)$ with a focal length of $120 \mathrm{~mm}$. The variable aperture stop in front of the mirror can define an illumination numerical aperture ranging from 0.002 to 0.015 . The beam is folded by a flat mirror $\left(\mathrm{M}_{2}\right)$ and hits the sample at a $6^{\circ}$ angle of incidence. The two mirrors are coated with Mo-Si multilayer optimized for maximum EUV reflection at each specific incidence angle. The reflected and diffracted light is then captured by an EUV CCD at a distance of 62 $\mathrm{mm}$. The CCD (Princeton Instuments MTE2) has $2048 \times 2048$ pixels with $13.5 \mu \mathrm{m}$ size and operates in vacuum. The sample is mounted on a XY stage with a travel range of $200 \mu \mathrm{m}$ along both axes and an accuracy of $\pm 5 \mathrm{~nm}$. The whole system is hosted in a vacuum chamber and operates at $10^{-7}$ mbar. RESCAN is installed at the XIL-II beamline of the Swiss Light Source synchrotron. The beamline is equipped with a monochromator with a bandwidth $\lambda / \Delta \lambda=1500$, which provides a beam with a temporal coherence suitable for CDI applications.

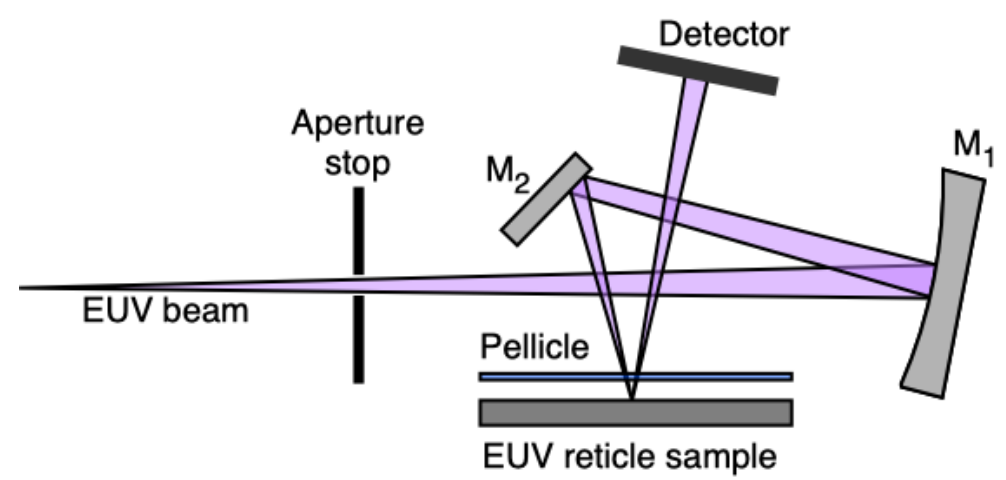

Figure 1. Optical layout of RESCAN. The EUV beam is focused by a toroidal mirror $\left(\mathrm{M}_{1}\right)$. The beam is folded by a flat mirror $\left(\mathrm{M}_{2}\right)$ onto the sample with a $6^{\circ}$ incidence angle. The reflected and diffracted light is captured by an EUV CCD at a distance of $62 \mathrm{~mm}$ from the sample plane.

Defect inspection in RESCAN is performed comparing the reconstructed image of an area of the mask to the image of another region with the same pattern (die-to-die mode) or to an aerial image calculated from the mask layout (die-to-database mode).

\section{EUV PHOTOMASKS DEFECT INSPECTION}

The standard EUV photomask works in reflection mode because EUV is largely absorbed by most materials. It consist of a low-thermal expansion material substrate with a chromium coating on the back and a Mo/Si multilayer on top. The multilayer is designed to ensure maximum reflectance for a wavelength of $13.5 \mathrm{~nm}$ and an incidence angle range of about 0 to 12 degrees to match the illumination angle of EUV scanners. ${ }^{11}$ The multilayer is capped with a $2-\mathrm{nm} \mathrm{Ru}$ protecting layer and the mask is patterned with a $70 \mathrm{~nm}$ TaBN absorber layer as shown in figure 2. A critical mask defect produces a modification in the printed wafer that may cause the final device to malfunction. Defects can be generated by errors in the absorber pattern (fig. 2A), by fall-on particles ${ }^{12}$ (fig. 2C) or by alterations in the substrate (fig. 2B). To mitigate the effect of fall-on particles, a transparent 
pellicle is installed 2 to $3 \mathrm{~mm}$ above the mask surface ${ }^{13-15}$ a particle has much less effect on the mask image if it is far away from the object plane (fig. 2D). ${ }^{16}$ The presence of the pellicle constitutes an additional challenge for conventional inspection and review tools that require the presence of optical components close to the mask surface and rule out the majority of inspection techniques employing non EUV wavelengths.

In addition to avoiding complex and expensive optical systems, CDI allows the direct investigation of phase defects originating from the substrate, it is generally compatible with through-pellicle inspection and can provide valuable information about the pellicle surface itself. ${ }^{17}$

RESCAN has been developed as a platform to demonstrate the use of CDI for EUV mask metrology and was successfully used to inspect samples with programmed amplitude and phase defects, and to perform the first through-pellicle EUV mask inspection test. ${ }^{17}$
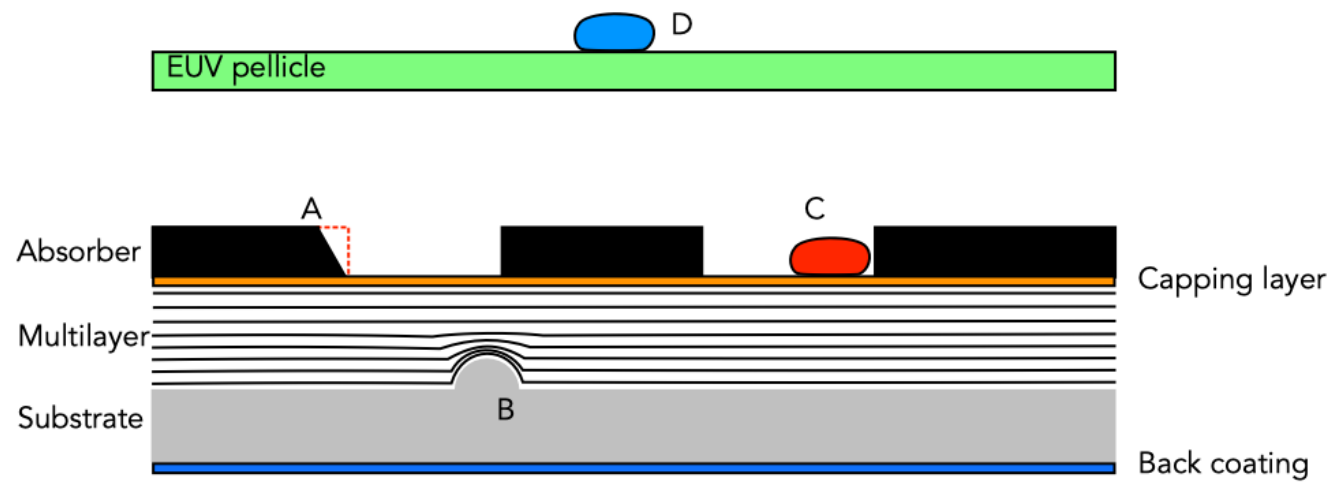

Figure 2. Structure of the EUV photomask. The mask consists of a low thermal expansion material substrate with a chromium back coating. The top surface is coated with a Mo/Si multilayer capped with a Ru protective layer and it is patterned by e-beam lithography with a TaBN absorber layer. The mask is equiped with a protective pellicle to mitigate the contamination from fall-on particles. Mask defects can originate from errors in the pattern (A), from substrate pits or bumps (B) and from fall-on particles (C). Large particles $(>6 \mu \mathrm{m})$ on the pellicle surface (D) can also cause errors in the printed pattern.

\subsection{Amplitude defects}

To test the capability of RESCAN to detect amplitude defects (fig 2A,C), we manufactured a a random-pattern mask sample with features similar to the ones present in a logic chip (fig. 3A) and we included a region with programmed defects with sizes ranging from $200 \mathrm{~nm}$ to $20 \mathrm{~nm}$ (fig. 3B).

The imaging resolution limit of RESCAN depends on the numerical aperture defined by the detector size and its distance from the sample. For this experiment, we selected a configuration with a resolution limit of $42 \mathrm{~nm}$. We calculated a reference image from a defect-free mask layout (fig. 3D) and we compared it with the reconstructed image of the sample (fig. 3C). The results, outlined in fig. 3E, show that we can detect defects down to a size of $50 \times 50 \mathrm{~nm}^{2} .^{18}$

\subsection{Phase defects}

Imperfections in the substrate surface or particles embedded in the multilayer may not be visible by scanning electron microscopy or atomic force microscopy, as the multilayer deposition can smooth them out almost completely, however, since the EUV beam penetrates the multilayer stack, these defects can generate a phase disturbance in the reflected light and distort the image of the mask. CDI is an ideal tool to detect and characterize phase defects since the phase information of the sample is a byproduct of the image reconstruction procedure.

To test the sensitivity of RESCAN to phase defects, we generated a mask sample with arrays of 200-nm and 50-nm diameter carbon pillars buried under the multilayer. ${ }^{18}$ An absorber random pattern similar to the one shown in figure $3 \mathrm{~A}$ was overlayed to the phase defect array. The heights of the carbon pillars were chosen to be $3.5 \mathrm{~nm}$ and $7.8 \mathrm{~nm}$, close to $\lambda / 4$ and $\lambda / 2$ respectively, in order to investigate the case of maximum and minimum 


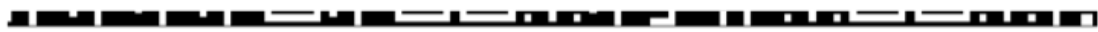

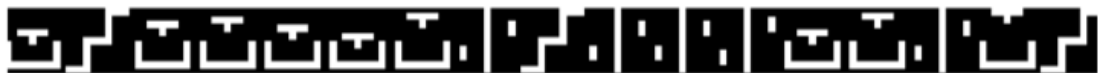

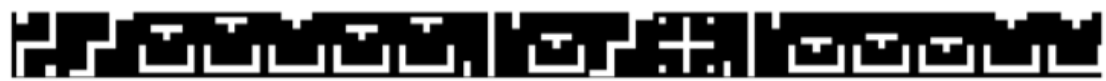
| |

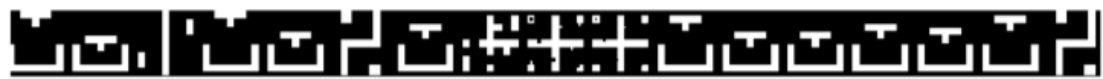

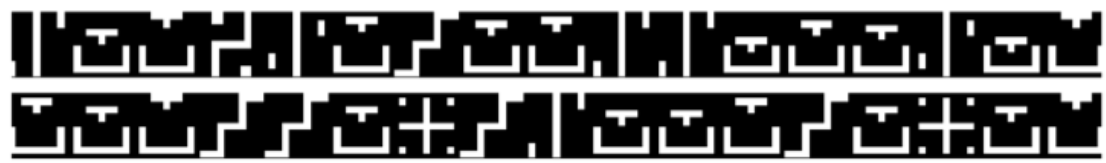

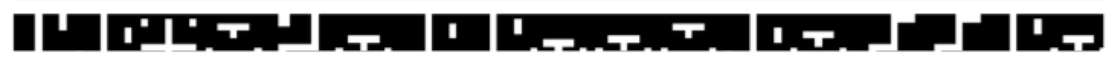

C

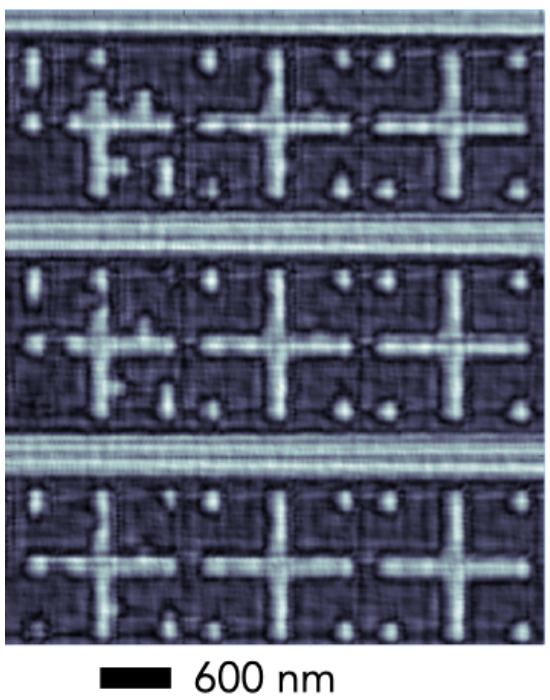

D

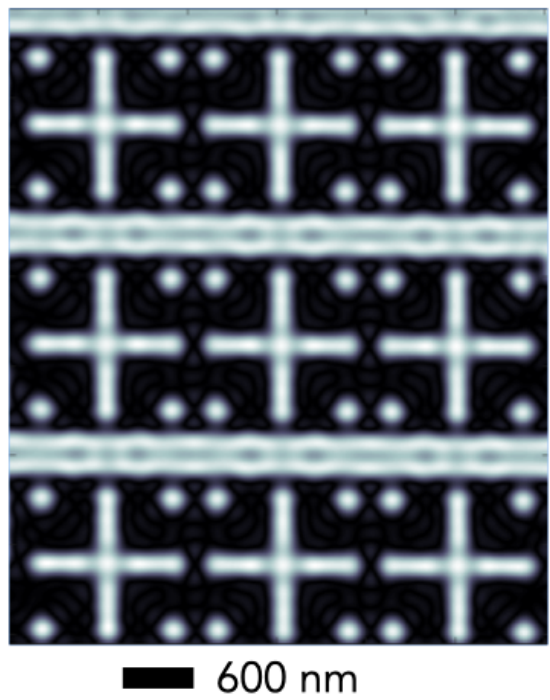

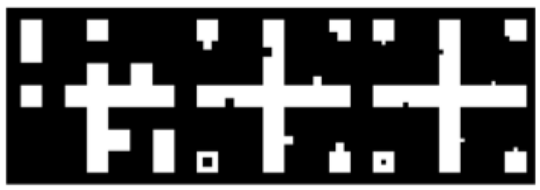
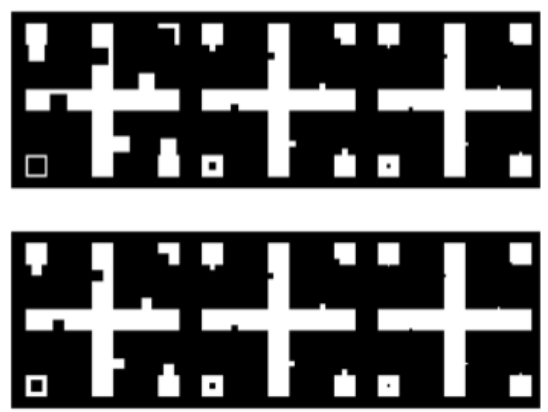

E

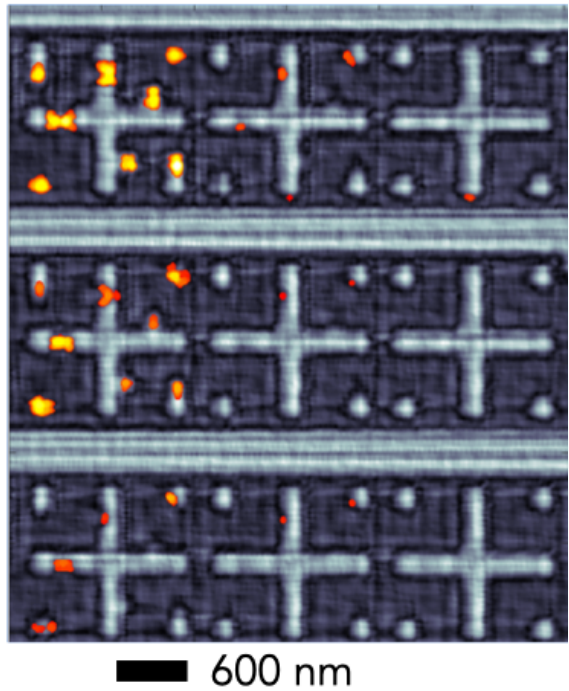

Figure 3. Experimental evaluation of the sensitivity of RESCAN to absorber defects. A. Layout of the sample. B. Detail of the programmed defect area with programmed absorber defects ranging from $200 \mathrm{~nm}$ (top right) to $20 \mathrm{~nm}$ (bottom left). C. Sample image reconstructed with RESCAN. D. Sample aerial image calculated from the defect-free design. E. Defect map obtained comparing $\mathrm{C}$ and $\mathrm{D}$. The defect signal is shown in color. Image modified from $\left.{ }^{18}\right]$.

phase contrast. Since we are working in a reflection geometry with a $6^{\circ}$ angle of incidence, the phase induced by a programmed bump defect of height $h$ should be:

$$
\Delta \phi=\frac{4 \pi h}{\lambda \cos \theta},
$$

however, in this case, the phase is not directly related to the topography of the sample, because the beam penetrates into the multilayer. To make an accurate prediction of the phase in the presence of a defect of a given shape, a model of the multilayer growth is required. Using such a model for 200 nm defects with a height of $3.5 \mathrm{~nm}$, we predicted a peak-to-valley phase variation of $0.96 \pi$ and we measured an average phase variation of $(0.95 \pm 0.02) \pi$ for the defects on the sample.

A detail of the reconstructed complex amplitude map is shown in fig. 4 where the color scale represents the phase and the intensity scale represents the magnitude of the image. The phase defects are clearly visible as red dots in the phase map. 

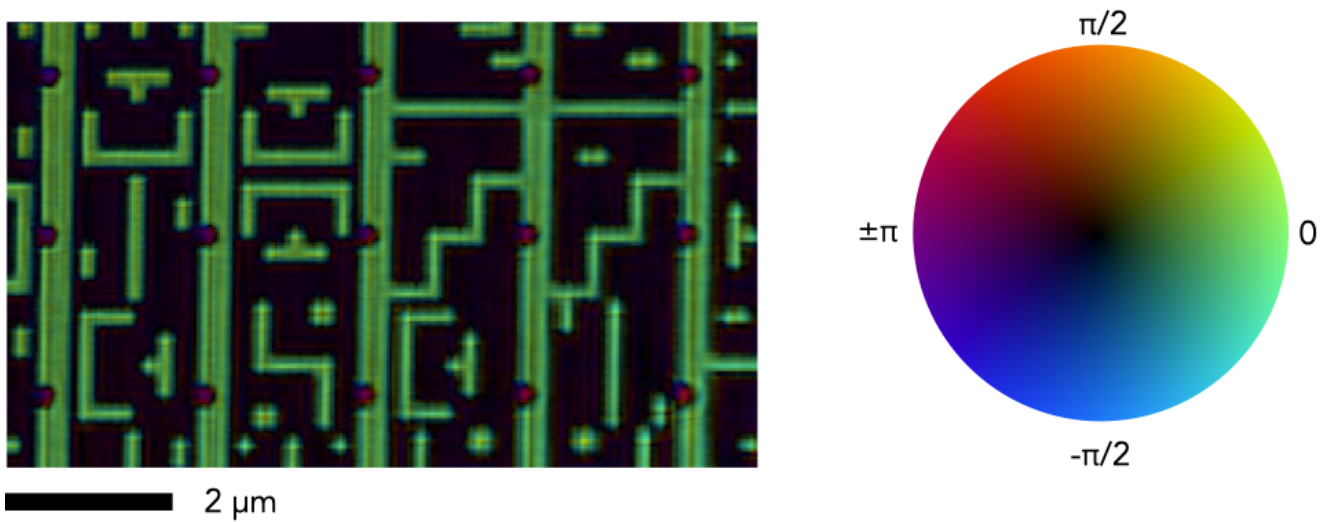

Figure 4. Reconstructed complex amplitude map of a sample with a buried array of carbon pillars with $200 \mathrm{~nm}$ diameter and $3.5 \mathrm{~nm}$ height.

\section{CONCLUSIONS}

The presence of defects on EUV photomasks for semiconductor lithography can impact the production of semiconductor devices. EUV mask inspection and review is an important tool to mitigate this risk. We investigated the possibility to use a lensless approach for EUV mask metrology as an alternative to traditional inspection methods. RESCAN is a synchrotron-based lensless EUV microscope developed for this purpose. Using RESCAN, we demonstrated the possibility to use CDI to inspect amplitude and phase programmed defects as small as $50 \times 50 \mathrm{~nm}^{2}$ and we outlined the potential of lensless imaging for semiconductor metrology applications.

\section{ACKNOWLEDGMENTS}

The authors wish to thank Markus Kropf and Michaela Vockenhuber for the technical support with the RESCAN end station and the XIL-II beamline at the Swiss Light Source and Serhiy Danylyuk from RWTH Aachen University for providing the model of the multilayer growth.

\section{REFERENCES}

[1] Mochi, I., Goldberg, K. A., La Fontaine, B., Tchikoulaeva, A., and Holfeld, C., "Actinic imaging of native and programmed defects on a full-field mask," Proc.SPIE 7636 (2010). doi:10.1117/12.846670.

[2] Mangat, P., Verduijn, E., Wood, O. R., Benk, M. P., Wojdyla, A., and Goldberg, K. A., "Mask blank defect printability comparison using optical and SEM mask and wafer inspection and bright field actinic mask imaging," Proc.SPIE 9658 (July 2015). doi:10.1117/12.2201048.

[3] Capelli, R., Dietzel, M., Hellweg, D., Koch, M., Kersteen, G., Gwosch, K., and Pagel, D., "Actinic metrology platform for defect review and mask qualification: flexibility and performance," Proc.SPIE 10957 (2019). doi:10.1117/12.2518596.

[4] Harada, T., Kishimoto, J., Watanabe, T., Kinoshita, H., and Lee, D., "Mask observation results using a coherent extreme ultraviolet scattering microscope at NewSUBARU," Journal of Vacuum Science \& Technology B: Microelectronics and Nanometer Structures Processing, Measurement, and Phenomena 27(6), 3203-3207 (2009). [doi:10.1116/1.3258633].

[5] Fienup, J. R., "Phase retrieval algorithms: a comparison," Appl. Opt. 21, 2758-2769 (Aug 1982).

[6] Thibault, P., Dierolf, M., Bunk, O., Menzel, A., and Pfeiffer, F., "Probe retrieval in ptychographic coherent diffractive imaging," Ultramicroscopy 109(4), 338 - 343 (2009).

[7] Li, P. and Maiden, A. M., "Ten implementations of ptychography," Journal of Microscopy 269(3), 187-194 (2018). doi:10.1111/jmi.12614. 
[8] Odstrčil, M., Menzel, A., and Guizar-Sicairos, M., "Iterative least-squares solver for generalized maximumlikelihood ptychography," Opt. Express 26, 3108-3123 (Feb 2018). doi:10.1364/OE.26.003108.

[9] Maiden, A. M. and Rodenburg, J. M., "An improved ptychographical phase retrieval algorithm for diffractive imaging," Ultramicroscopy 109(10), 1256 - 1262 (2009). doi:10.1016/j.ultramic.2009.05.012.

[10] Maiden, A., Johnson, D., and Li, P., "Further improvements to the ptychographical iterative engine," Optica 4, 736-745 (Jul 2017). doi:10.1364/OPTICA.4.000736.

[11] van Schoot, J., van Setten, E., Troost, K., Bornebroek, F., van Ballegoij, R., Lok, S., Stoeldraijer, J., Finders, J., Graeupner, P., Zimmermann, J., Kuerz, P., Pieters, M., and Kaiser, W., "High-NA EUV lithography exposure tool progress," Proc.SPIE 10957 (2019). doi:10.1117/12.2515205.

[12] Hyun, Y., Kim, J., Kim, K., Koo, S., Kim, S., Kim, Y., Lim, C., and Kwak, N., "EUV mask particle adders during scanner exposure," Proc.SPIE 9422 (2015). doi:10.1117/12.2085626.

[13] Hong, J., Park, C., Lee, C., Nam, K., Jang, Y., Wi, S., and Ahn, J., "Development of full-size EUV pellicle with thermal emission layer coating," Proc.SPIE 10809 (2018). doi:10.1117/12.2501772.

[14] Timmermans, M. Y., Mariano, M., Pollentier, I., Richard, O., C, H., and Gallagher, E. E., "Free-standing carbon nanotube films for extreme ultraviolet pellicle application," Journal of Micro/Nanolithography, MEMS, and MOEMS 17 (2018). [doi:10.1117/1.JMM.17.4.043504].

[15] Gallagher, E., Timmermans, M. Y., Pollentier, I., Lee, J., Mariano, M., Adelmann, C., Huyghebaert, C., Scholze, F., and Laubis, C., "CNTs in the context of EUV pellicle history," in [Advanced Lithography : Extreme Ultraviolet (EUV) Lithography IX], Proc.SPIE 10583, 105831E (2018). [doi:10.1117/12.2297710].

[16] No, H.-R., Lee, S.-G., Oh, S.-H., and Oh, H.-K., "Pattern degradation with larger particles on EUV pellicle," Proc.SPIE 10809 (2018). doi:10.1117/12.2502784.

[17] Mochi, I., Timmermans, M. Y., Gallagher, E. E., Mariano, M., Pollentier, I., Rajendran, R., Helfenstein, P., Fernandez, S., Kazazis, D., and Ekinci, Y., "Experimental evaluation of the impact of carbon nanotube euv pellicles on reticle imaging," Journal of Micro/Nanolithography, MEMS, and MOEMS 18(1), $1-7-7$ (2019). doi:10.1117/1.JMM.18.1.014002.

[18] Mochi, I., Fernandez, S., Nebling, R., Locans, U., Helfenstein, P., Rajeev, R., Dejkameh, A., Kazazis, D., Tseng, L.-T., and Ekinci, Y., "Absorber and phase defect inspection on euv reticles using RESCAN," Proc.SPIE 10957 (2019). doi:10.1117/12.2515160. 